\title{
Chromosomal aberrations in transitional cell carcinoma that are predictive of disease outcome are independent of polyploidy
}

\author{
J.M.S. BARTLETT, L. ADIE, A.D. WATTERS, J.J. GOING* and K.M. GRIGOR† \\ University Departments of Surgery and *Pathology, Glasgow Royal Infirmary, Glasgow, and †University Department of Pathology, \\ University of Edinburgh, Edinburgh, Scotland, UK
}

\begin{abstract}
Objective To determine whether aneusomy for chromosomes 7, 9 and 17 (reported to predict recurrence in up to $65 \%$ of patients with superficial transitional cell bladder cancer and thus providing the opportunity for early and effective treatment) reflects specific genetic events on these chromosomes or merely wider unspecific genetic damage to the cell, e.g. that increased copy numbers for 7 and 17 reflect tumour polyploidy.

Materials and methods The study comprised 25 primary tumours; $6 \mu \mathrm{m}$ thick sections from formalin-fixed and paraffin-embedded tumours were analysed. Chromosome copy numbers were determined by fluorescence in situ hybridization (FISH) using pericentromeric probes for chromosomes 7, 8, 9, 10, 11 and 17. A minimum of 200 nuclei per tumour area were scored by two independent observers.
\end{abstract}

Results Eight of the 25 tumours examined (32\%) showed no evidence of chromosomal abnormalities as detected by FISH for any chromosomes analysed. Twelve tumours $(48 \%)$ showed abnormalities for one or two chromosomes, five tumours $(20 \%)$ showed abnormalities for multiple chromosomes and one tumour showed abnormalities for all chromosomes analysed, suggestive of polyploidy.

Conclusions Chromosomal abnormalities predictive of recurrence occur largely in the absence of other gross chromosomal lesions. In a small proportion of cases other chromosomes are affected, but this is almost always distinct from tumour polyploidy.

Keywords Bladder cancer, recurrence, molecular diagnosis, fluorescence in situ hybridization, chromosomes $9,7,17$, polyploidy

\section{Introduction}

The diagnosis of bladder cancer relies heavily on direct cystoscopic examination of the bladder which enables the surgeon to biopsy and resect tumours [1,2]. Such resection is curative for $\approx 20 \%$ of patients, but this success is limited to patients with noninvasive or only locally invasive primary TCC (pTa/pT1). About one in five patients present with advanced disease (pT2-4) at diagnosis [1,2]. Overall, $70 \%$ of patients presenting with TCC are destined to have recurrence, the risk of which is a function of time, although most patients will have recurrence within 2-4 years of diagnosis. Uniquely amongst cancers, patients who experience one recurrence almost inevitably return with further episodes of recurrent disease such that the patient requires frequent hospitalization to monitor disease recurrences [1,2]. Furthermore, a significant proportion of patients with recurrent disease will develop aggressive detrusor muscle invasive tumours (10-20\% of patients [1,2]). The high

Accepted for publication 1 June 1999 frequency of recurrence and risk of progression make frequent cystoscopic monitoring of patients a requirement of disease management $[1,2]$.

Recurrence rates can be reduced markedly by instillation chemotherapy at initial diagnosis [3], but until recently no mechanism has been available to discriminate between patients at risk of recurrence and those for whom such chemotherapy would be unwarranted. As a result many urologists prefer to defer chemotherapy until recurrence is detected (Scottish Urological Oncology Group, personal communications) to avoid over-treating patients who do not recur and exposing such patients to the genotoxic affects of chemotherapy without cause.

Molecular markers which predict recurrence have been detected by our previous research [4-6] and identify patients most likely to benefit from regular cystoscopic surveillance and/or instillation chemotherapy at primary diagnosis. Abnormalities of chromosomes 7, 9 and 17 have been shown to predict recurrence in up to $65 \%$ of patients at primary diagnosis [7]. The challenge is to expand these findings and increase the efficiency of such tests before their acceptance into clinical diagnostic 
medicine. To achieve this goal it is first necessary to determine the nature of the genetic alterations detected.

The development and progression of tumours, including TCC, is driven by an accumulation of genetic abnormalities [8-15]. Whilst chromosome-specific abnormalities are frequently described, polyploidy is also a frequent event in tumours and TCC is no exception [12-14]. The evaluation of panels of two or three chromosomal markers may fail to detect tumour polyploidy and lead to a misunderstanding of the tumour genetic profile. We have therefore expanded our previous studies of chromosomes 7,9 and 17 to determine whether numerical aberrations occurred as a result of or independently from tumour polyploidy. We have further studied 25 of the 54 primary tumours from patients with TCC that either recurred (32) or did not (22), described previously [4-7], a proportion of whom also progressed to detrusor muscle invasion (11). Fluorescence in situ hybridization (FISH) data for chromosomes 7, 9 and 17 were available for all 54 tumours; FISH analysis for chromosomes 8, 10 and 11 was performed on the 25 primary tumours selected from these groups and quantitative analysis of chromosome copy number obtained.

\section{Materials and methods}

The 25 primary tumours selected for this retrospective study represent the spectrum of genetic alterations seen in the previous study (monosomy chromosome 9, aneusomy chromosomes 7 and 17. and apparent normosomy). The pathology for all tumours was reviewed following the UICC 1978 guidelines [16].

Morphologically normal disomic urinary bladder epithelium served as a quality control for the FISH method. Both normal tissues and tumours were accessed from participating pathology laboratories (Departments of Pathology at Glasgow and Edinburgh Royal Infirmaries) and serial sections of the formalin-fixed, paraffin waxembedded tissues were cut onto silane-coated slides for analysis. All tumours analysed had a representative section stained with haematoxylin and eosin (H\&E), and were re-staged and re-graded by one specialist pathologist (K.M.G.) following the UICC 1978 guidelines [16].

Dual-target FISH was performed with satellite repeat sequence DNA probes for chromosomes 7, 8, 9, 10, 11 and 17 , as described previously $[4,5]$. Hybridization was visualized with fluorescein isothiocyanate (FITC) avidin, biotinylated anti-avidin and then the signal-amplified with an additional FITC-avidin step (Vector Laboratories, Burlingame, CA) for biotinylated probes, and with sheep antidioxigenin (Boehringer Mannheim, Lewes, East Sussex, UK), FITC donkey antisheep (Stratech Scientific,
Luton, Beds, UK) for digoxigenin-labelled probes. This protocol yielded easily quantifiable results of equal colour intensity for all probes.

The signals were visualized using an epifluorescence microscope (Leica, Milton Keynes, UK), with a 100-W mercury arc lamp, and photomicrography performed using a Wild 48/52 photoautomat system (Leica, UK). Regions for analysis by FISH were identified by examining H\&E-stained slides and areas to be scored marked on an enlarged photocopy (200\%) of the section. Signals for each chromosome in at least 200 discrete nuclei in control and carcinoma sections were counted using a multichannel counter. The number of signals $(0-8)$ were recorded. One to three tumour areas were analysed, and a proportion by three independent observers.

As a measure of overall chromosomal copy number, the mean chromosomal copy number $(\mathrm{MCCN})$ was computed as the total number of hybridization signals divided by the total number of nuclei. Monosomy was assessed by criteria previously defined [4]. Normal ranges for MCCN and monosomy were defined as the mean \pm 3 SD. Polysomy was defined as tumours with $\geqslant 10 \%$ of cells with $\geqslant 3$ signals per nucleus [12-14]. The mean result from all tumour areas (2-3 per tumour) was calculated to give an overall value for each tumour event.

\section{Results}

\section{FISH}

For chromosomes 7, 9 and 17, our previous reports indicated that $24 \%$ of primary tumours (13 of 54) showed loss of one copy of chromosome 9 [1]; 19\% of primary tumours (10 of 54) showed increased copy numbers for either chromosome 7 or 17 or both [5-7] and one patient also had an increased copy number of chromosome 9 . Only $3.7 \%$ of primary tumours showed simultaneous loss of chromosome 9 and gain of chromosomes 7 and 17 [5-7]. Both monosomy of chromosome $9(P=0.0016)$ and polysomy of 7 and $17(P=0.0095)$ were significantly associated with tumour recurrence, being almost exclusively detected in primary tumours from patients who exhibited recurrence as described previously [4-7].

For chromosomes 8, 10 and 11, in 25 selected primary tumours from our previous study, 32\% (eight of 25) showed no abnormalities in any of the chromosomes tested, either in this study or previously (chromosomes 7-11 and 17, data not shown). Nine of the remaining 17 primary tumours had one abnormality (Table 1). Of these, five were monosomic for chromosome 9 whilst normal for all other chromosomes evaluated, two were polysomic for chromosome 17 and two were newly identified as polysomic for chromosome 8 . 
Table 1 Mean chromosomal copy numbers for aneusomic tumours in patients with one or multiple chromosomes affected, shown in bold

\begin{tabular}{|c|c|c|c|c|c|c|}
\hline \multirow{2}{*}{$\begin{array}{l}\text { Stage/ } \\
\text { grade }\end{array}$} & \multicolumn{6}{|c|}{ Mean chromosomal copy numbers for chromosome } \\
\hline & 7 & 8 & 9 & 10 & 11 & 17 \\
\hline \multicolumn{7}{|l|}{ Single } \\
\hline pTaG1 & 1.68 & 1.7 & 0.83 & 1.54 & 1.68 & 1.67 \\
\hline pTaG1 & 1.61 & 1.71 & 1.03 & 1.62 & 1.7 & 1.67 \\
\hline pTaG2 & 1.56 & 1.71 & 1.05 & 1.72 & 1.68 & 1.64 \\
\hline pTaG2 & 1.67 & 1.69 & 1.85 & 1.76 & 1.74 & 2.12 \\
\hline pTaG2 & 1.61 & 2.54 & 1.60 & 1.65 & 1.71 & 1.64 \\
\hline pT1G2 & 1.84 & 1.58 & 0.87 & nd & nd & 1.87 \\
\hline pT1G2 & 1.82 & 1.79 & 1.81 & nd & nd & 2.13 \\
\hline pT1G3 & 1.74 & 1.6 & 1.18 & 1.68 & 1.74 & 1.8 \\
\hline pT2G3 & 1.76 & 2.64 & 1.37 & nd & nd & 1.78 \\
\hline \multicolumn{7}{|l|}{ Multiple } \\
\hline pTaG1 & 1.61 & 2.05 & 0.84 & 1.73 & nd & 1.60 \\
\hline pTaG1 & 1.60 & nd & 0.87 & 0.86 & 1.67 & 1.53 \\
\hline pTaG1 & 2.33 & 2.34 & 1.11 & 1.60 & 2.28 & 2.44 \\
\hline pTaG2 & 1.66 & 1.71 & 0.91 & 1.57 & 1.72 & 2.16 \\
\hline pT1G2 & 1.98 & 2.48 & 1.66 & 1.35 & 1.85 & 2.01 \\
\hline pT1G3 & 2.79 & 3.31 & 2.25 & 2.37 & 2.5 & 2.71 \\
\hline pT1G3 & 2.71 & 2.54 & 1.68 & 2.07 & 2.31 & 2.54 \\
\hline pT1G3 & 2.21 & 2.44 & 1.59 & nd & 2.24 & 2.33 \\
\hline
\end{tabular}

nd, not done.
Four tumours had abnormalities in two or three chromosomes; of these, three were monosomic for chromosome 9 (Table 1). Additional lesions were polysomy 17 , polysomy 8 or monosomy 10 (one case each). One tumour showed polysomy for chromosomes 7, 8 and 17 with no alterations in chromosomes 9,10 or 11 (Table 1), although the copy number for chromosome 10 was at the low end of the normal range.

Only four cases had abnormalities in more than three chromosomes; of these, one was polysomic for all six chromosomes tested (Table 1), suggesting a possible polyploidy. Of the remaining tumours, two were normal for chromosome 9 but polysomic for all other chromosomes tested, and one was polysomic for chromosomes 7, 8, 11 and 17, monosomic for chromosome 9 and normal for chromosome 10 .

\section{Stage and grade}

Of the 25 tumours analysed for all chromosomes, 15 were pTa, nine pT1 and one pT2, and nine were grade 1 , nine grade 2 and seven grade 3 . Of the 15 pTa tumours, six showed no chromosomal abnormalities, five showed alterations of single chromosomes and four showed multiple aneusomic events. Of the $10 \mathrm{pT} 1 / 2$ tumours, two showed no abnormalities, three showed alterations of single chromosomes (including the pT2 tumour) and five showed alterations of multiple chromosomes.
Of the G1 tumours, four showed no chromosomal abnormalities, two showed monosomy 9 and three showed multiple alterations (maximum five chromosomes). Of G2 tumours, three showed no chromosomal abnormalities, four showed alterations affecting single chromosomes and two showed alterations involving multiple chromosomes (up to 3). Of G3 tumours, one showed no alterations, three showed aneusomy of one chromosome and three showed aneusomy of multiple chromosomes.

\section{Discussion}

In our recent studies we identified aneusomy of chromosomes 9,7 and 17 as potential risk factors for recurrence in TCC of the urinary bladder [4-7]. Monosomy for chromosome 9 or polysomy for chromosomes 7 and 17 were associated significantly with subsequent disease recurrence in a retrospective cohort analysis [4-7]. As it is possible that these changes reflect wider genetic damage such as tumour polyploidy we extended the studies to encompass chromosomes infrequently involved in genetic alterations in TCC (chromosomes 10 and 11) and included analysis of chromosome 8. Abnormalities in chromosomes 8, 10 and 11 are more frequently associated with disease progression.

The results presented here suggest that: (i) monosomy 9 occurs independently other chromosomal abnormalities; (ii) polysomy of chromosomes 7 and 17 appears, in 
most cases, to occur in tandem, as in renal cell cancers [17], but may also be associated with other chromosomal changes; (iii) these lesions are rarely associated with tumour polyploidy.

Monosomy 9 occurred independently of other lesions, although in some tumours abnormalities of other chromosomes were identified; half of the present tumours investigated showed monosomy 9 alone. In the other tumours, monosomy 9 was identified in the presence of polysomy 8,7 or 7 and 17, and monosomy 10. No clear pattern emerged to associate changes in chromosome 9 with other chromosome abnormalities. This finding suggests that loss of chromosome 9 does not require other gross chromosomal changes, nor does it necessarily drive further gross chromosomal alterations.

In $>80 \%$ of cases both chromosomes 7 and 17 were polysomic, to a similar degree as that reported previously for renal cell cancers [17]. From the evidence presented here, excluding polyploidy in these tumours and showing this event to be independent of monosomy 9, the present findings are evidence for a true association between aneusomy of chromosomes 7 and 17 . The causal mechanisms behind such a parallel alteration in two discrete chromosomes are at present unclear. In a small proportion of tumours, polysomy 17 appears in isolation, which may indicate that polysomy 17 is the initial event in the subsequent duplication of both chromosomes, but mechanisms which might explain this process are at present unknown.

Whilst chromosome 17 was gained in association with gain in chromosome 7 in many cases, in most such cases there was aneusomy of other chromosomes (notably chromosomes 8 and 11), which compounds the issues underlying this important genetic change.

High stage and grade are associated with increased genetic instability [8-10] but in the present investigation, confined to primary tumours, there was no clear association between increased stage or grade and increasing chromosomal abnormality in these few cases. Similar proportions of tumours in the pTa and pT1, and G1 vs G2 vs G3 groups were normosomic, aneusomic for one chromosome or aneusomic for multiple chromosomes. The exclusion by design of high-stage tumours from the present study may have masked changes associated with such tumours.

Tumour polyploidy is a risk factor for disease progression [18]. However, in this study, whilst in one tumour all chromosomes showed similar increases in copy number, suggesting polyploidy, in all other tumours at least one chromosome had a normal copy number, suggesting that these tumours were not truly polyploid. Our previous reports have shown that aneusomy of chromosomes 9 and 17 in primary superficial TCC are significantly linked to subsequent tumour recurrence
[4-7]. However, neither lesion is significantly associated with progression to higher tumour stage [4-7]. It would appear that these events are linked with superficial or locally invasive (pTa/pT1) tumour recurrence, therefore we proposed that genes on these chromosomes are specifically involved in the process of tumour recurrence, as distinct from tumour invasion [4-7].

In conclusion, following our recent studies identifying genetic variables predictive of recurrence, the present results exclude tumour polyploidy as the underlying genetic event and show that aneusomy of chromosome 9 or chromosomes 7 and 17 are discrete genetic events. The linkage between these events and tumour recurrence suggests that genes involved in tumour recurrence in the absence of clinical progression are located on these candidate chromosomes, as previously hypothesized.

\section{Acknowledgements}

This Research was funded by a grant from the Scottish Office Home \& Health Department No: C2263 and was presented at the Pathology Society of the United Kingdom, 1-3 July 1998 and in part at the British Association for Cancer Research 24-27 June 1998.

\section{References}

1 Kirk D. Improving the management of superficial bladder cancer. Br Med J 1993; 306: 1014-5

2 Hall RR, Parmar MKB, Richards AB, Smith PH. Proposals for changes in cystoscopic follow up of patients with bladder cancer and adjuvant intravesical chemotherapy. Br Med J 1994; 304: 257-60

3 Tolley DA, Parmar MKB, Grigor KM et al. The effect of intravesical mitomycin $\mathrm{C}$ on recurrence of newly diagnosed superficial bladder cancer: a further report with 7 years followup. J Urol 1996; 155: 1233-8

4 Bartlett JMS, Watters AD, Ballantyne SA et al. Is chromosome 9 loss a marker of disease recurrence in TCC of the bladder? Br J Cancer 1998; 77: 2193-8

5 Watters AD, Ballantyne SA, Going JJ, Grigor KM, Bartlett JMS. Aneusomy of chromosomes 7 and 17 predicts recurrence of transitional cell carcinoma of the urinary bladder. BJU Int 1999; in press

6 Watters AD, Ballantyne SA, Going JJ, Grigor KM, Cooke TG, Bartlett JMS. Chromosomes $7 \& 17$ as predictors of disease recurrence and progression in transitional cell carcinoma of the urinary bladder. Br J Cancer 1998; 78: 75

7 Bartlett JMS, Watters AD, Ballantyne SA, Going JJ, Grigor KM. Molecular diagnosis of recurrence by analysis of primary TCC. J Pathol 1998; 186: A12

8 Saran KK, Gould D, Godec CJ, Verma. RS. Genetics of bladder cancer. J Mol Med 1996; 74: 441-5

9 Sandberg AA, Berger CS. Review of chromosome studies in urological tumours. II. Cytogenetics and molecular genetics of bladder cancer. J Urol 1994; 51: 545-60 
10 Knowles MA. Molecular genetics of bladder cancer. $\mathrm{Br}$ J Urol 1995; 75: 57-66

11 Kallioniemi A, Kallioniemi OP, Citro G et al. Identification of gains and losses of DNA sequences in primary bladder cancer by comparative genomic hybridization. Genes Chromosomes Cancer 1995; 12: 213-9

12 Waldman FM, Carroll PR, Kerschmann R, Cohen MB, Field FG, Mayall BH. Centromeric copy number of chromosome 7 is strongly correlated with tumour grade and labelling index in human bladder cancer. Cancer Res 1991; 51: 3807-13

13 Sauter G, Moch H, Carroll P, Kerschman R, Mihatsch MJ, Waldman FM. Chromosome 9 loss detected by fluorescence in situ hybdridization in bladder cancer. Int J Cancer 1995; 64: 99-103

14 Matsuyama H, Bergerheim USR, Nilsson I et al. Nonrandom numerical aberrations of chromosomes 7, 9, and 10 in DNA-diploid bladder cancer. Cancer Genetics and Cytogenetics 1994; 77: 118-24

15 Sandberg AA. Chromosome changes in early bladder neoplasms. J Cell Biochem 1992; 50: 76-9

16 UICC TNM classification of malignant tumours. Geneva: UICC, 1978
17 Corless CL, Aburatani H, Fletcher JA, Houseman DE, Amin $\mathrm{MB}$, Weinberg DS. Papillary renal cell carcinoma quantitation of chromosomes 7 and 17 by FISH, analysis of chromosome 3p for LOH, and DNA ploidy. Diag Mol Pathol 1996; 5: 53-64

18 Neulander E, Kaneti J, Chaimovitz C, Sion-Vardy N, Douvdevani A, Soloway MS. Deoxyribonucleic acid ploidy and the clinical pattern of grade 2 superficial bladder cancer. J d'Urol 1997; 157: 1254-9

\section{Authors}

J.M.S. Bartlett, PhD, Senior Lecturer.

L. Adie, BSc.

A.D. Watters, BSc.

J.J. Going, PhD.

K.M. Grigor, MD.

Correspondence: Dr J.M.S. Bartlett, University Department of Surgery, Level II, Queen Elizabeth Building, Glasgow Royal Infirmary, Glasgow, G31 2ER, Scotland, UK.

e-mail: gqva20@udcf.gla.ac.uk 